\title{
Compulsory community and involuntary outpatient treatment for people with severe mental disorders.
}

KENNEDY, C.

2019

This is an original manuscript / preprint of an article published by Taylor \& Francis in Issues in Mental Health Nursing on 22/4/2019, available online: http://www.tandfonline.com/10.1080/01612840.2019.1600359. 


\title{
() \\ Cochrane \\ Nursing Care
}

The Mission of the Cochrane Nursing Care Field (CNCF) is to improve health outcomes through increasing the use of the Cochrane Library and supporting Cochrane's role by providing an evidence base for nurses and related healthcare professionals involved in delivering, leading or researching nursing care. The CNCF produces 'Cochrane Corner' columns (summaries of recent nursing-care-relevant Cochrane Reviews) that are regularly published in collaborating nursing-care-related journals. Information on the processes this Field has developed can be accessed at: http://cncf.cochrane.org/evidence-transfer-program-review-summaries

\author{
Cochrane Nursing Care Field - Cochrane Review Summary
}

Prepared for the

\section{Issues in Mental Health Nursing}

\section{TITLE: Compulsory community and involuntary outpatient treatment for people with severe mental disorders}

Cochrane Corner Writer:

Catriona Kennedy PhD, RN, DN

QNIS Professor of Community Nursing

School of Nursing and Midwifery

Robert Gordon University

Aberdeen, Scotland

c.m.kennedy1@rgu.ac.uk

A member of the Cochrane Nursing Care (CNC) 


\section{Background:}

An increasing number of countries are using compulsory community treatment (CCT) for people with severe mental health problems. This approach aims to keep people at home and out of hospitals but requires them to adhere to treatment and follow up appointment schedules. If they fail to adhere, then healthcare professionals can return them to in-patient care on a compulsory basis.

The enduring nature of mental health problems often necessitates repeated and cyclical admissions to hospital. Many countries are reforming their services to ensure people can manage their health problems close to home. CCT reflects the growing shift to community rather than in-patient care in mental health and features in countries including the United Kingdom (UK), Australia, Canada, Israel and New Zealand.

There exits opposing views as to the utility of CCT. Those supporting this approach view it as a more person/family focused approach to compulsory treatment. Allowing people to stay amongst family and friends and thereby maintaining control over aspects of their life may contribute to a more stable life pattern. Opponents of CCT see it as a potentially powerful approach which might impact on the relationship between the person with mental health problems and the healthcare professional. They contend that CCT may emphasise the control held by healthcare professionals. This in turn may lead to mistrust and potentially drive people away from statutory services rather than towards them in times of need.

\section{Objective/s:}

The overall aim was to establish the benefits, effectiveness or possible hazards of compulsory treatment and the specific objective of this review was to 'examine the effectiveness of compulsory community treatment for people with severe mental illness' through CCT (of any form) with standard voluntary care and where possible compare different types of CCT' (Kisely, Campbell and O'Reilly 2017).

Participants in randomised controlled trials were adults with severe mental illness who were managed in the community. Studies, which included both substance abuse and severe mental illness, were eligible for inclusion but substance abuse alone was 
not. Three comparisons were identified as relevant; court ordered outpatient commitment compared with entirely voluntary care; CCT versus supervised discharge and community treatment orders versus standard care.

\section{Intervention/Methods:}

CCT varies across international jurisdictions. For example in the UK and parts of the USA and Canada it is classified as extended leave or supervised practice and gives healthcare professionals the rights to send a person with severe mental illness back to hospital if they do not adhere to treatment and follow up schedules. These rights apply on discharge from hospital to the community. Community treatment orders (CTO) apply in Australia and Canada and give healthcare professionals greater power as they can apply an order whether the person is in hospital or not.

Regardless, СCT gives considerable power and decision making to healthcare professionals. It is therefore important to assess the evidence as to the benefits, effectiveness or potential hazards/adverse implications of CCT.

\section{Results:}

Three studies (two from the USA and one from the UK) were included which involved a total of 749 adult participants. Two trials compared CCT versus standard care or voluntary care and the third trial compared a form of СTO to supervised discharge. This review did not provide evidence of effectiveness of CCT against standard voluntary care in any of the main outcome areas; health service use, costs, social functioning, mental state, quality of life or satisfaction with care.

The reviewers contend that for those receiving CCT there is some evidence (from one trial) that they were less likely to be victims of violent or non-violent crime. Furthermore the findings indicate that short periods of conditional leave may be as effective (or non-effective) as compulsory treatment in the community.

\section{Conclusions:}

The authors of this review were unable to draw any firm conclusions as to the effects of CCT on any of their main outcomes over standard care. The three trials were of low to medium quality therefore can offer little guidance for nurses working with 
people who have severe mental health problems and their families. Furthermore no evidence was found that compulsory treatment was more or less effective than periods of conditional leave. This is important as it emphasises the need to reflect on key components of the nurse patient relationship. Being aware that implementation of a CCT order could be perceived as putting a power imbalance in place reminds nurses of the importance of excellent and open communication with those they work with. Exploring patient and family preferences and choices and ensuring they understand the treatment and follow up plan is critical to helping adherence to the benefit of those affected.

\section{Implications for Practice:}

Encouraging and supporting people with severe mental illness at home to adhere to treatment and follow up schedules is known to be challenging. The findings of this review suggest that СCT has little positive impact on outcomes for patients but nor does it increase the use of power and coercion. Therefore nurses should view use of CCT orders cautiously and standard care should be recommended.

As part of the multidisciplinary team nurses working with people with severe mental illness in the community have a critical role to play in supporting them and their families to live a fulfilled and stable life at home and in avoiding hospital admissions. Given the lack of evidence around effectiveness of CCT, impeccable assessment which includes a clear and honest dialogue with the patient and family is essential. Preferences for support need to be explored, jointly agreed and revisited frequently as part of an ongoing dialogue. Involving patients and families as partners in care can maximise the potential for a robust nurse, patient and family relationship which helps adherence to treatment plans thus increasing the chances of sustaining people at home rather than frequent readmissions to hospital.

\section{References:}

Kisely SR, Campbell LA, O'Reilly R. Compulsory community and involuntary outpatient treatment for people with severe mental disorders. Cochrane Database of 
Systematic Reviews 2017, Issue 3. Art. No.: CD004408. DOI:10.1002/14651858.CD004408.pub5. 